\title{
An Uncommon Cause of Diarrhoea and the Importance of an Internist Approach
}

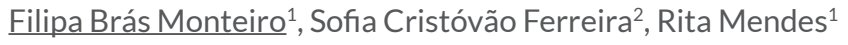 \\ ${ }^{1}$ Hospital Egas Moniz, Centro Hospitalar Lisboa Ocidental, Lisbon, Portugal \\ ${ }^{2}$ Hospital Egas Moniz, Centro Hospitalar Lisboa Ocidental, Instituto Português de Oncologia, Lisbon, Portugal
}

How to cite this article: Brás Monteiro F, Cristóvão Ferreira S, Mendes R. An uncommon cause of diarrhoea and the importance of an internist approach. EJCRIM 2016;3: doi:10.12890/2016_000501.

Conflicts of Interests: The Authors declare that there are no competing interests.

This article is licensed under a Commons Attribution Non-Commercial 4.0 License

\section{ABSTRACT}

Background: Human intestinal spirochetosis is a condition defined by the presence of spirochetes attached to the colonic epithelium. Brachyspira aalborgi and Brachyspira pilosicoli may cause the disease in humans.

Case report: We describe the case of a 58-year-old patient who presented with epigastric abdominal pain and diarrhoea. He was thought to be having a myocardial infarction and underwent an angiogram with subsequent stenting of the circumflex coronary artery. However, the pain and diarrhoea were still present after the procedure and the patient now had sepsis. An exploratory laparotomy was inconclusive. The patient improved on intravenous antibiotics and was discharged, but returned to the emergency department 2 days later with the same complaints. He was then admitted to an internal medicine ward where the diagnosis of intestinal spirochetosis was made. The patient was started on metronidazol and completed a 10-day antibiotic course with full recovery of his symptoms.

Conclusion: This case highlights the importance of an internist-based approach that could have prevented two invasive procedures and the accompanying risks.

\section{LEARNING POINTS}

- Uncommon causes of diarrhoea are a diagnostic challenge.

- Errors of clinical judgement can lead to invasive and potentially harmful procedures.

- An internist-based approach can prevent diagnostic mistakes and be in the patient's best interests.

\section{KEYWORDS}

Diarrhoea, Brachyspira aalborgi, type 2 myocardial infarction, peritonitis

\section{INTRODUCTION}

Human intestinal spirochetosis is a condition defined by the presence of spirochetes attached to the colonic epithelium, first described in $1967^{[1]}$. The Gram-negative bacteria Brachyspira aalborgi and Brachyspira pilosicoli may cause the disease in humans. As it is more common in underdeveloped countries, when diagnosed in the western world, it is usually associated with immunocompromised states, namely, HIV infection ${ }^{[2]}$. The diagnosis can be made by histology, since collections of spirochetes on the luminal side of the epithelium can be seen on colonic biopsy specimens. The spirochetosis can be confirmed by polymerase chain reaction or detection of serum anti-Brachyspira antibodies ${ }^{[3]}$. The main clinical finding is aqueous diarrhoea, and although there is no specific therapy, most patients recover after treatment with the antibiotic metronidazole ${ }^{[4]}$. Here, we report a case of spirochetosis with Brachyspira aalborgi in an immunocompetent man. 


\section{CASE DESCRIPTION}

We describe the case of a 58-year-old patient, who was a smoker (80 packs per year) and known to have hypertension but was not on any medication.

He presented with diarrhoea and chest/epigastric pain evolving over the previous 24 hours. He was seen at home by the medical emergency team and the ECG showed Q waves in infero-lateral leads and an ST elevation ( $<2 \mathrm{~mm})$ in anterior leads. This was assumed to be a myocardial infarction (MI) with symptoms presenting over the previous 24 hours. The angiogram revealed a narrowed circumflex artery ( $90 \%$ stenosis, TIMI 2) and a completely occluded right coronary artery (RCA) with filling being possible via left anterior descendant (LAD) collaterals. Two drug-eluting stents were placed in the circumflex artery and the decision to intervene on the RCA was delayed until after reversible ischaemia studies were carried out.

The patient was placed on double anti-platelet therapy (DAPT) and the post-procedure echocardiography showed a hypertrophic left ventricle (LV) with posterior and inferior akinesia and lateral hypokinesia and moderate LV systolic dysfunction.

Blood tests revealed a very minor troponin I increase (from $0.16 \mu \mathrm{g} / \mathrm{dl}$ to $0.29 \mu \mathrm{g} / \mathrm{dl} ; \mathrm{N}<0.02 \mu \mathrm{g} / \mathrm{dl}$ ) with significant renal dysfunction (AKIN 3; creatinine $3.8 \mathrm{mg} / \mathrm{dl}$; N 0.7-1.2 mg/dl), liver cytolysis and elevation of inflammatory markers (CRP increased from $50.2 \mathrm{mg} / \mathrm{dl}$ to $65 \mathrm{mg} / \mathrm{dl}$; $\mathrm{N}<0.5 \mathrm{mg} / \mathrm{dl})$.

The patient was septic (HR $115 \mathrm{bpm}, \mathrm{BP} 85 / 63 \mathrm{mmHg}$, temperature $39.2^{\circ} \mathrm{C}$, lactate 3.4 ) at that point and kept complaining of epigastric pain and loose stools (more than 15 times a day). After discussing the patient with general surgery personnel, abdominal computed tomography was performed revealing an enlarged bowel with no obstruction point identified. It was decided to operate on the patient the next day although he was on DAPT. No obstruction was seen during surgery despite remarkable small bowel distension. A primary peritonitis was assumed based on a cloudy abdominal fluid and the patient was started on ceftriaxone and total parenteral nutrition (TPN). Over the next few days, the abdominal pain resolved, but the loose stools continued and culture results (blood, urine, stools and abdominal fluid) were negative. Enteral feeding was attempted and tolerated on the fifth day after surgery. However, 10 days after surgery the abdominal pain worsened and the inflammatory markers rose again (CRP $28 \mathrm{mg} / \mathrm{dl}$ ). Antibiotic therapy was empirically escalated to meropenem and vancomycin since no isolation had been possible until then. The patient was continued on both antibiotics for 10 days and then was discharged, as he was clinically improved, with a final diagnosis of type $2 \mathrm{Ml}$ and peritonitis.

However, the patient was readmitted to the emergency department after 2 days with worsening diarrhoea (more than 15 times a day) and abdominal pain. The patient had noticed a decrease in urinary output over the previous 2 days. On examination, he was pale and dehydrated, with tenderness on the left lower quadrant.

Blood tests revealed increasing inflammatory markers with an elevated CRP (from $26 \mathrm{mg} / \mathrm{dl}$ to $34 \mathrm{mg} / \mathrm{dl}$; $\mathrm{N}<0.5 \mathrm{mg} / \mathrm{dl}$ ) and a decrease in renal function as he had been discharged with creatinine values within the normal range (creatinine $2.52 \mathrm{mg} / \mathrm{dl} ; \mathrm{N} \mathrm{0.7-1.2} \mathrm{mg/dl).}$

An abdominal CT was repeated revealing an enlarged small bowel but no other changes (Fig. 1).

Meropenem was restarted and despite the improvement in blood test results, the patient maintained watery diarrhoea and abdominal discomfort.

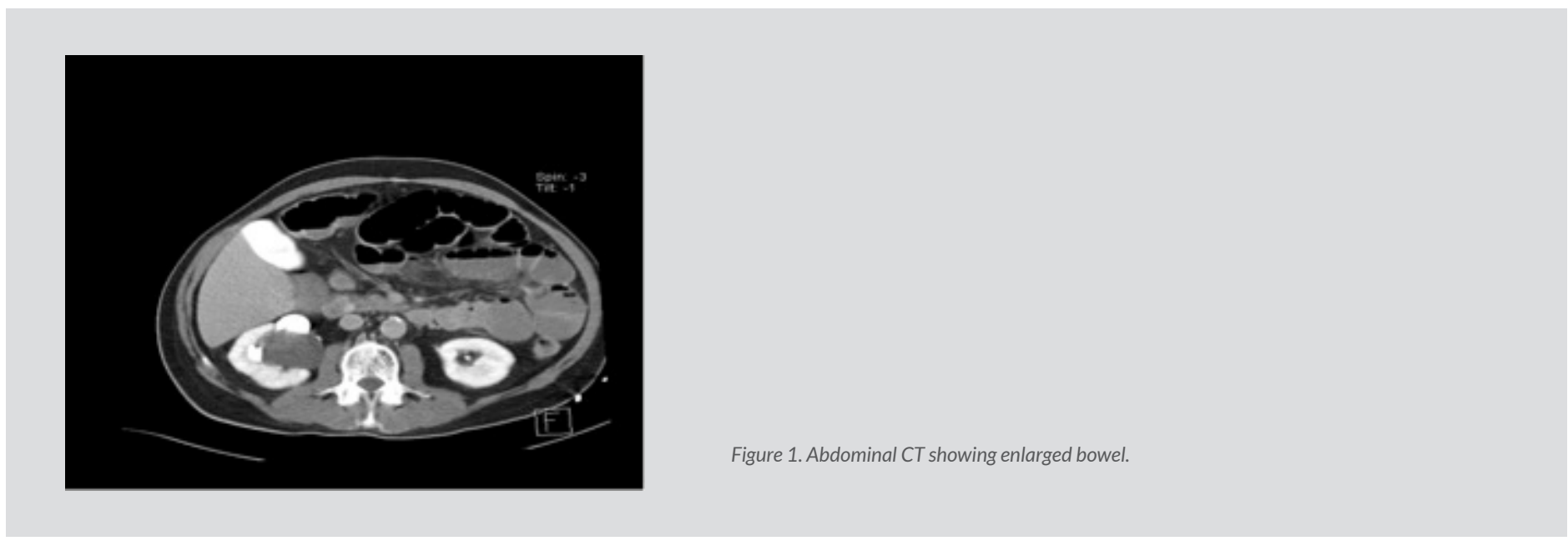


All blood cultures, stool exams, including tests for Clostridium difficile, Giardia and Entamoeba, were negative. The patient had no epidemiological context, no recent travel history or sexual risk behaviours as a heterosexual man.

At this point the patient had had diarrhoea for over 4 weeks and had already undergone an extremely invasive investigation as a laparotomy had been performed less than 1 month previously while he was on DAPT given the recent angioplasty.

A rectosigmoidoscopy revealed focal hyperaemic areas, suggesting Kaposi's sarcoma despite a negative HIV test (Figs. 2 and 3).

Biopsy results a couple of days later revealing infiltration with rod spiral forms adhesive to the enteral surface, suggesting spirochetosis due to Brachyspira aalborgi (Figs. 4 and 5).

The patient was then placed on metronidazol $500 \mathrm{mg}$ four times a day for 10 days with complete resolution of the abdominal pain, diarrhoea and inflammatory markers. He was discharged 4 days later by which time blood tests had completely normalized. He was seen in clinic 1 month later and confirmed complete resolution of symptoms with no diarrhoea or loose stools.

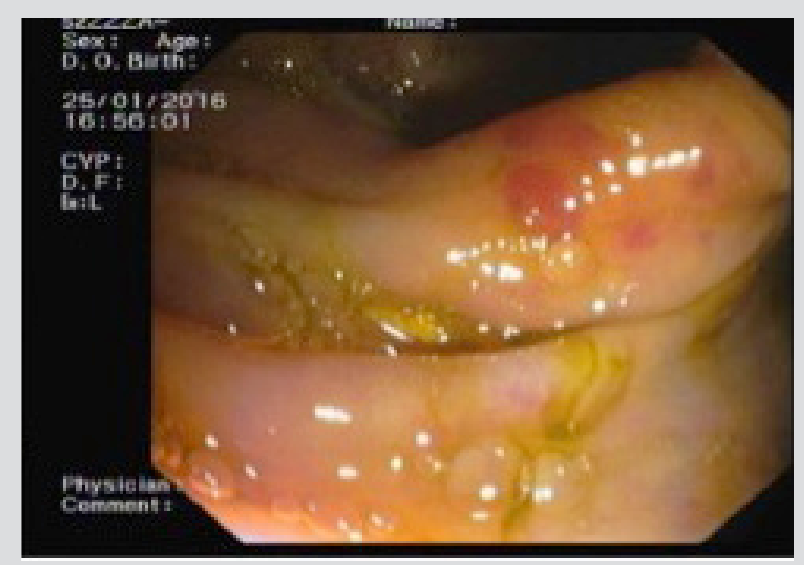

Figure 2. Violet-coloured hyperaemic areas seen on rectosigmoidoscopy

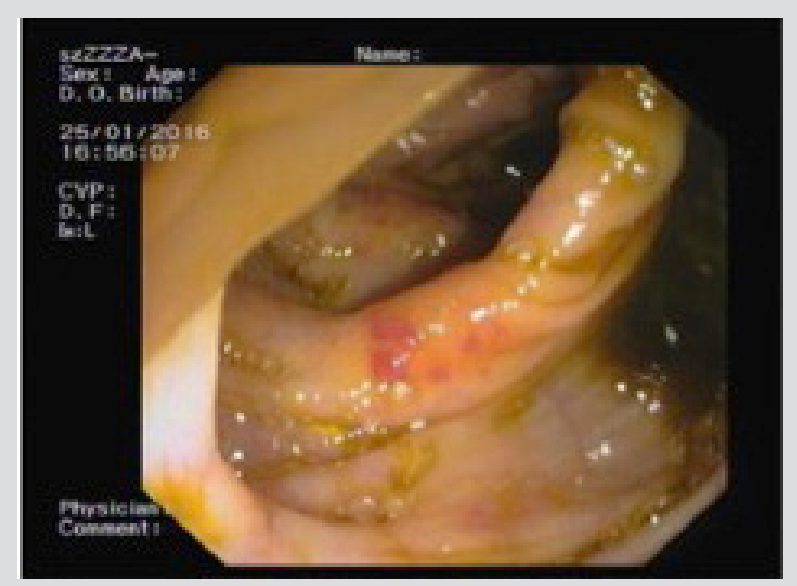

Figure 3. Violet-coloured hyperaemic areas seen on rectosigmoidoscopy

\section{DISCUSSION}

This case report is particularly important because it describes a rare cause of chronic diarrhoea in an immunocompetent patient who underwent several invasive procedures including an exploratory laparotomy while on DAPT. The patient was also admitted to hospital twice and was placed on three different antibiotics over a 1-month period.

With this report, we hope to raise awareness of infrequent causes of chronic diarrhoea that are sometimes overlooked in developed countries since they are more common in other parts of the world or in patients with the appropriate epidemiological context ${ }^{[5]}$ as opposed to the case we are describing.

It is also relevant that the diagnosis was made through an internal medicine-based approach, since the patient was initially admitted to a cardiology ward and was only reviewed by cardiology and general surgery teams, with the result that several invasive and potentially unnecessary procedures were carried out. 

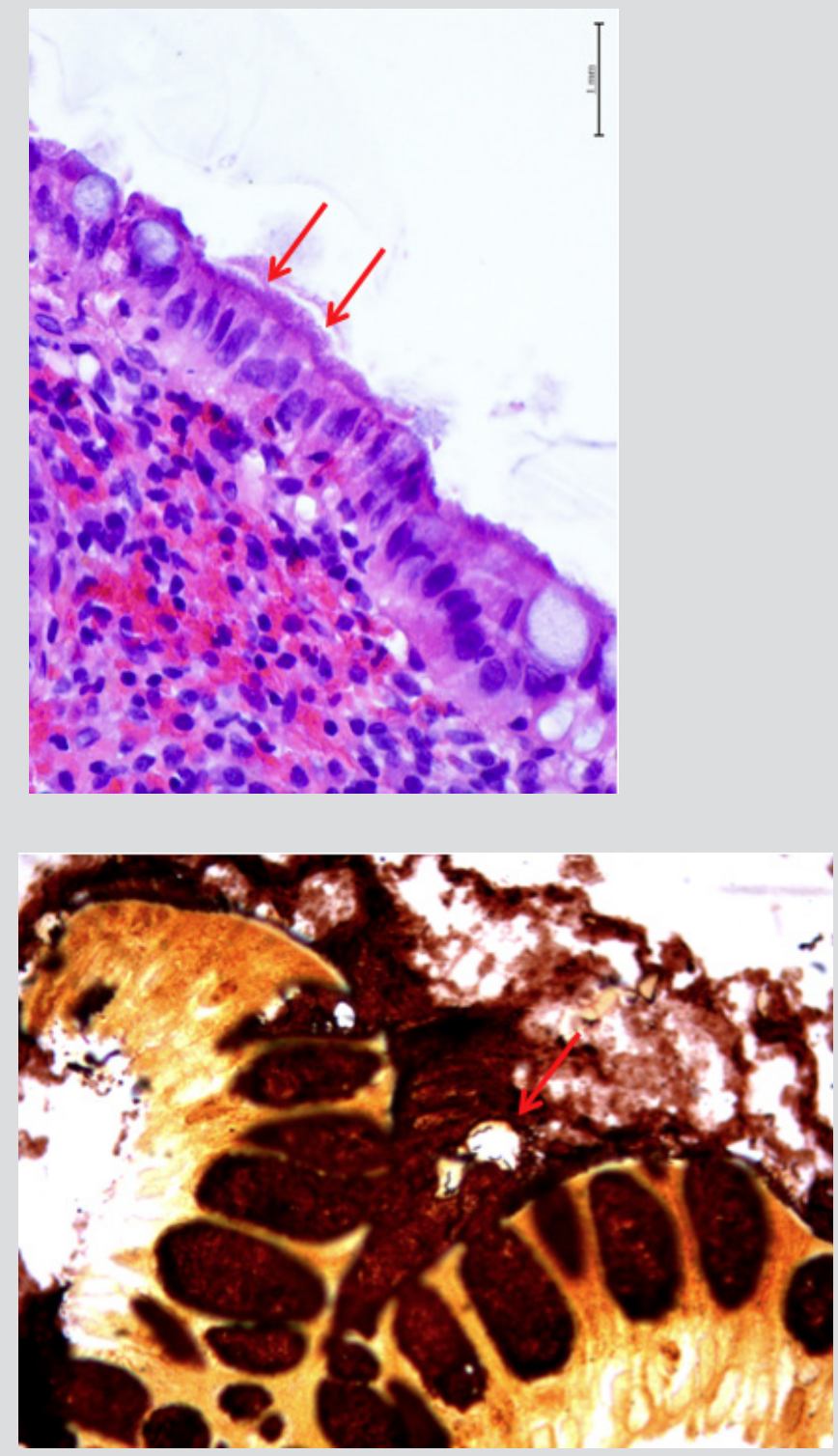

\section{REFERENCES}

1. Harland WA, Lee FD. Intestinal spirochaetosis. BMJ 1967;3:718-719.

2. Tateishi Y, Takahashi M, Horiguchi S, Funata N, Koizumi K, Okudela K, et al. Clinicopathologic study of intestinal spirochetosis in Japan with special reference to human immunodeficiency virus infection status and species types: analysis of 5265 consecutive colorectal biopsies. BMC Infect Dis 2015;15:13.

3. Iwamoto J, Ogata S, Honda A, Saito Y, Murakami M, Ikegami T, et al. Human intestinal spirochaetosis in two ulcerative colitis patients. Intern Med 2014;53:2067-2071.

4. Weisheit B, Bethke B, Stolte M. Human intestinal spirochetosis: analysis of the symptoms of 209 patients. Scand J Gastroenterol 2007;42:1422-1427.

5. Efstathia T, Jan-Olaf G. Human intestinal spirochetosis - a review. Ger Med Sci 2010;8:Doc01. 